\title{
Should medical curricula include greater training on the detection and reporting of child abuse?
}

\begin{abstract}
Reports of child abuse and neglect are increasing in prevalence globally. This increase has been observed in the United States and worldwide and is believed that many cases of child abuse and neglect go undetected and unreported in the USA and abroad. Practicing physicians and their supporting medical professional staff including nurses, medical assistants, and mid-level providers are in a pivotal position to assess, document, initiate and report suspected child abuse and neglect (SCAN) during initial physical assessments, where the suspected injuries may easily be documented during such physical examinations.

Medical students typically complete up to two years of basic medical sciences in their academic training, during which standard core courses in anatomy, physiology, pathology, physical diagnosis and introduction to clinical medicine among others are included in the curriculum. This is followed by two years of clinical training in core clinical specialties including mentor supervised experiences in family and internal medicine, surgery, obstetrics and gynecology, psychiatry, pediatrics and elective specialties and options for research experiences are included. Most medical curricula are already intense, with little room for the inclusion or introduction of additional modules. Decades ago, medical curricula faced the same issue while attempting to find pathways to incorporate additional topics including nutrition in the medical curricula, despite the now wide recognition that syndromes of over- and under- nutrition play a vital role in the pathogenesis and sequelae of many common disorders, and in their recovery. In many areas of undergraduate medical education, the time constraints in curriculum implementation may be even more acute, with little available time or resources available to address such topics as nutrition education or incorporation of the common topics included in SCAN. ${ }^{1,2}$
\end{abstract}

To address this critical topic, students entering their clinical years (typically the third and fourth years) of the medical education seem unprepared to detect, assess, and report SCAN. It is not the fault of the student doctor that they may feel unprepared, as the large if not extraordinary volume of medical knowledge they are expected to have mastered by this juncture in their medical education is virtually overwhelming to many students. In addition, introduction to the rigors of the clinical environment they are expected to maneuver through also requires familiarity with numerous clinical procedures, for which they may have only gained rudimentary knowledge of during their basic sciences experience. In addition, instead the health care and legal systems they are about to embark into have yet to reach a universal and cooperative protocol to assess and procedures to discover and report SCAN; and the medical education system has yet to mandate and integrate SCAN awareness, assessment, and reporting into the medical curriculum, particularly prior to medical students entering clinical rotations.

This deficiency in the preparation of medical students can lead to more unreported cases of SCAN and more children left as victims of repeated child abuse and neglect. The purpose of this paper is to bring renewed awareness of the deficiency in medical students' knowledge of and training in SCAN, as well as provide them a foundation in the presentation of child abuse and neglect in the clinical setting, and engender a brighter, more positive and secure outcome for those unfortunate children who may inadvertently fall prey to such behavior.
Volume 12 Issue 5 - 202I

\section{Athena Ivanoff, Orien L Tulp}

Department of Science Arts and Technology, Montserrat and the University of Health and Humanities, USA

Correspondence: Athena Ivanoff, Department of Science Arts and Technology, Montserrat and the University of Health and Humanities,Virgin Islands, USA, Email athena.ivanoff@usat.edu

Received: October 21, 2021 | Published: October 27, 2021

\section{Acknowledgments}

None.

\section{Conflicts of interest}

The authors declare that there are no conflicts of interest.

\section{Funding}

None.

\section{References}

1. Ivanoff AE A Medical Student Primer on Detecting and Reporting Suspected Child Abuse and Neglect (SCAN). EC Paediatrics. 2019; (2019):907-912.

2. Ivanoff AE, Einstein GP, Tulp OL. A Review for Medical Students and Student Doctors on Detecting and Reporting Suspected Child Abuse and Neglect (SCAN) from Clinical and Forensic Observations. Abstract R7600, Proceedings, EB 2020, FASEB J. 2020;35(1). 